\title{
Sensitivity of Different Collagens to Proteolytic Enzyme Treatment
}

\author{
Anokhova V.D. ${ }^{1}$ Chupakhin E.G. ${ }^{1}$ Pershina N.A. ${ }^{2}$ Storublevtsev S.A. ${ }^{3}$ Antipova L.V. ${ }^{3}$ \\ Matskova L.V. ${ }^{1}$ Antipov S.S. ${ }^{1,3,4, *}$ \\ ${ }^{1}$ Immanuel Kant Baltic Federal University, 2, Universitetskaya str., Kaliningrad, Russia, 236000, Russia \\ ${ }^{2}$ Voronezh State University, 1, Universitetskaya pl., Voronezh, 394018, Russia \\ ${ }^{3}$ Voronezh State University of Engineering Technology, 19, Revolution Ave., Voronezh, 394036, Russia \\ ${ }^{4}$ Moscow State University of Technologies and Management, 73, Zemlyanoy Val, Moscow, 109004, Russia \\ *Corresponding author. Email: ss.antipov@gmail.
}

\begin{abstract}
Collagen is a ubiquitously expressed essential structural component of all metazoan extracellular matrices. It is present in organisms ranging from sponges to humans. Although the general functions of collagens are comparable, their characteristics are quite distinct. There are 19 different types of animal collagens, as defined by their tissue location. Much less is known about the structure of collagens from hydrations as compared to mammal collagens. This research aimed to compare the sensitivity of two types of Collagen; one is from hydration (silver carp, Hypophthalmichthys molitrix) and other is from mammals - (cattle, Bos taurus Taurus) to trypsin, collagenase and acidic proteases. Here we report the differential sensitivity of these two collagens to proteases and the different patterns produced by proteolytic digestion. We also show that it is possible to isolate Collagen from fish skin according to our protocol, as the purified substance is specifically cleaved by collagenase. We also show the different sensitivities of fish and cattle collagen to proteases.
\end{abstract}

Keywords: collagen, animal collagens, proteolytic enzyme

\section{INTRODUCTION}

Collagen is an essential component of the extracellular matrix in all multicellular organisms as it serves to stabilize tissues of animals, fish, and birds $[1,2]$. Collagen mediates bio-absorption (absorption of inorganic components), provides hygroscopic properties to tissues (retention of water molecules), participates in cell adhesion (attachment of cells to the intercellular matrix mediated by their membrane receptors), facilitates cell proliferation and migration, as well as many other functions [3]. Native mammalian collagen molecules are a form of fibrillar proteins which tend to assemble a large (up to several microns) triple helix protein structures. Organization of Collagen permits the creation of multivalent supramolecular networks and makes the collagen oligomers relatively resistant to proteases [4]. Mammalian collagen structure and the structure of its complexes are well known [5, 6]. Moreover, like cellulose, the basic structural component of plant cell walls, Collagen can swell in water due to a large number of functional groups with hydration ability [7, 8], a feature which makes Collagen a promising component perspective source for functional nutrition $[9,10]$.

Due to these are features, Collagen belongs to the natural materials, which have found wide applications is widely needed in medicine, cosmetology and personal hygiene [11]. At the same time, Collagen is found in the by-products and wastes of the processing industry for poultry, meat, and fish industries [12]. The sources of Collagen can be considered readily available and inexpensive, and their utilization for the production of collagen-based substances would reduce environmental pollution.

The hydration potential of hydration Collagen is even of more acute scientific and applied interest since the aquatic habitat of hydrations is likely to be reflected in the differences in the structure and functions of its Collagen [13]. About this question, there is still a lack of knowledge about many aspects of hydration Collagen, i.e. its sensitivity to proteases, hydration capability, sorption ability, especially concerning aromatic substances, and also its wound healing activity. Currently, there is no data on the fractional composition and modular structure of hydrations collagen, its 3D-structure and resistance to various types of enzymes. In this work, we aimed to identify the sensitivity of Collagen from the widely distributed aquatic organisms (Hypophthalmichthys molitrix (silver carp)) to trypsin, collagenase and a mixture of weakly acidic proteases. We compared proteolytic digestion products of hydration and mammalian collagens (Bos taurus (cattle)), by comparing the molecular weight profiles of proteolytic peptides from these two collagen types. 


\section{MATERIALS AND METHODS}

All reagents were used according to the manufacturer's instructions. Acetic acid (CAS 64-19-7), sodium chloride (NaCl) (CAS 7647-14-5), trichloroacetic acid (CAS 76-039), sodium dodecyl sulphate (SDS) (CAS 151-21-3), ammonium persulfate (CAS 7727-54-0), acetonitrile (7505-8), acrylamide (CAS 79-06-1), bis-acrylamide (CAS 110-26-9), sodium persulfate (CAS 7775-27-1), trypsin from bovine pancreas (CAS 9002-07-7), collagenase from Clostridium histolyticum and Coomassie Brilliant Blue R250 (CAS 6104-58-1) were purchased from Sigma Aldrich. Molecular weight markers were purchased from Bio-Rad. As a matrix and a calibration standard for the MALDI-TOF mass spectrometer, an $\alpha$-Cyano-4hydroxycinnamic acid (Bruker Daltonic) was used. All other chemicals and reagents used were of analytical grade, dissolved in MilliQ water if required.

A mixture of weakly acidic proteases was obtained from Aspergillus niger by clay cultivation (under aerobic conditions). The sample contained mainly peptidases and proteases. The enzymes were active in the $\mathrm{pH}$ range from 3.0 to 6.0 in temperatures ranging from $30^{\circ} \mathrm{C}$ to $58^{\circ} \mathrm{C}$. The optimum of enzyme activity was in the $\mathrm{pH}$ range from 3.5 to 4.5 and at temperatures from 45 up to $50^{\circ} \mathrm{C}$. The enzymes were inactivated at temperatures above $60^{\circ} \mathrm{C}$.

All mass-spectra data were acquired on the Autoflex MALDI-TOF mass spectrometer (Bruker Daltonic, Germany). MALDI-TOF settings were as follows: high voltage ion source 19.5 and $18.45 \mathrm{kV}$, the high voltage on first reflector $8 \mathrm{kV}$; laser: $\mathrm{Nd}$ : YAG, frequency $1 \mathrm{GHz}$, 500 shot.

Lyophilisation of Collagen was carried out in a freeze dryer Labconco Triad in standard mode.

\subsection{Sample collection and skin preparation}

The outer dermal layer of cattle and silver carp specimens were collected and stored at $-20{ }^{\circ} \mathrm{C}$ until use. The skin was removed from the samples, cut into small pieces $(0.5 \times 0.5 \mathrm{~cm})$, the residual subcutaneous tissue was manually removed from the skin. The skin pieces were used as a source for Collagen.

\subsubsection{Removal of non-collagenous proteins and fat from the skin}

Skin pieces were soaked in $0.2 \mathrm{M} \mathrm{KOH}$ solution at 1:10 (w/v, skin/solution ratio) for 1 day. The alkali solution was changed every day. Excess of $0.2 \mathrm{M} \mathrm{KOH}$ solution was washed off with distilled water. Fat from the skin pieces was removed by treatment with $10 \%$ isopropyl alcohol at $1: 10(\mathrm{w} / \mathrm{v})$ skin/solution ratio for $24 \mathrm{~h}$. Finally, samples were washed with an ample amount of distilled water and freeze-dried.
Collagen from cattle and silver carp skin was isolated as described previously [8]. The fish skins were descaled and hydrolyzed with a peroxide-alkaline mixture, for 1.0-1.5 hour at $20-25{ }^{\circ} \mathrm{C}$ to remove ballast proteins and allow swelling of the dermal layer. Subsequently, the Then, skin pieces were washed with water for 25 minutes at $12{ }^{\circ} \mathrm{C}$. Next, the skin samples were ground while centrifuged on a top spinning centrifuge with a tip diameter of $2 \mathrm{~mm}$ and soaked in $6 \%$ acetic acid at a ratio of $1: 3(\mathrm{w} / \mathrm{v})$ skin/solution. The resulting mixtures were ground on an emulsifier for 5 minutes, and fibres were separated away by stratification of the mixtures for 4-6 days. The mixtures were homogenized again during $7 \mathrm{~min}$, and solutions were filtered through a sieve with a $0.5 \mathrm{~mm}$ mesh diameter and lyophilized for storage.

\subsubsection{Isolation of collagen from the skin}

The lyophilized skin was soaked in $0.5 \mathrm{M}$ acetic acid at a 1:10 (w/v) skin/solution ratio for 3 days at $4{ }^{\circ} \mathrm{C}$ with gentle stirring. The mixture was centrifuged at $20,000 \mathrm{~g}$ for 60 min at $4{ }^{\circ} \mathrm{C}$. The supernatants, containing dissolved Collagen, were collected and kept at $4{ }^{\circ} \mathrm{C}$. The residual Collagen was re-extracted for 2 days from the same skin samples using the same conditions. The supernatants after two centrifugations were combined. The majority of ballast proteins was salted-out by adding crystalline $\mathrm{NaCl}$ to a final concentration of $0.9 \mathrm{M}$. After centrifugation to eliminate ballast proteins, Collagen was precipitated by adding $\mathrm{NaCl}$ to a final concentration of $2.3 \mathrm{M}$ in $0.05 \mathrm{M}$ Tris- $\mathrm{HCl}$ ( $\mathrm{pH}$ 7.5). The resultant precipitate, containing Collagen, was collected by centrifugation at $20.000 \mathrm{~g}$ for $30 \mathrm{~min}$ at $4{ }^{\circ} \mathrm{C}$ and then dissolved in 10 volumes of $0.5 \mathrm{M}$ acetic acid. The obtained solution was desalted by gelexclusion chromatography in a column with $5 \mathrm{~g}$ Biogel P30 carrier. After elution with $0.05 \mathrm{M}$ Tris- $\mathrm{HCl}(\mathrm{pH} 7.2)$, the desalted fractions of Collagen were analyzed using a MALDI-TOF Bruker spectrophotometer.

\subsection{Hydrolysis protocol}

Collagens from cattle and silver carp in Tris-HCl buffer pH 5.6 (50 ul) were treated with trypsin, collagenase and a mixture of weakly acidic proteases in Tris- $\mathrm{HCl}$ buffer $\mathrm{pH}$ 7.8 by a conventional protocol [14] during 10, 30, $60 \mathrm{~min}$ or overnight. Hydrolysis was stopped by adding 5 ul acetic acid to the reactions. An aliquot of $1 \mathrm{ul}$ of the reaction mixtures was placed on a MALDI platform, and after drying, the aliquotes were overlayed by with 1 ul of a concentrated matrix solution (a-Cyano-4-hydroxycinnamic acid). Samples were analyzed on the MALDI-TOF after crystallization of the samples. 


\subsection{Polyacrylamide gel electrophoresis (PAGE) analysis of collagen}

The patterns of products of proteolytic digestion of collagens were analyzed by polyacrylamide gel electrophoresis under a denaturing condition, in the presence of sodium dodecyl sulphate (SDS-PAGE) according to the classical Laemmli method [15]. Powdered collagens (ASC and PSC) were dissolved in $0.02 \mathrm{M}$ sodium phosphate buffer ( $\mathrm{pH} 7.2)$ containing $1 \%(\mathrm{w} / \mathrm{v})$ SDS and $3.5 \mathrm{M}$ urea at $1 \mathrm{mg} / \mathrm{mL}$ concentration. The samples were gently stirred at $4{ }^{\circ} \mathrm{C}$ for $2 \mathrm{~h}$ to dissolve Collagen.

\section{RESULTS}

Gel electrophoretic analysis of undigested collagenous fractions from silver carp and cattle demonstrated differences (Fig. 1A, lane N1 and N2, respectively). The presence of proteins with a molecular weight higher than $120 \mathrm{kDa}$ was characteristic for the silver carp collagen preparation (Fig. 1A, line N2). In contrast, the cattle collagen sample was enriched in the smaller molecular weight proteins (Figure 1A, line N1).

Next, we compared collagens from the different sources on SDS-PAAG after treatment with enzymes - trypsin (Figure 1B), collagenase (Figure 1A) and with a mixture of weakly acidic proteases (Figure 1C). Collagens from various sources (silver carp (Hypophthalmichthys molitrix) and cattle (Bos taurus Taurus)), although digested under similar conditions, gave different peptide profiles. Collagen from silver carp showed less sensitivity (more resistance) to trypsin as evidenced by the presence of higher molecular weight peptides after digestion as compared to cattle collagen (Figure 1B, lane 1 and 2, respectively). In contrast, Collagen from cattle showed less sensitivity to collagenase as compared to Collagen from silver carp (Figure 1A). Interestingly, treatment with a mixture of weakly acidic proteases had a similar effect on both collagen preparations (Figure 1C). We cannot fully explain this phenomenon yet. Perhaps this is due to the method of preparation of the mixture of weakly acidic proteases from Aspergillus nigger. However, we cannot exclude the possibility of increased the sensitivity of both types of Collagen to all or a set of weakly acidic proteases.
Collagenase

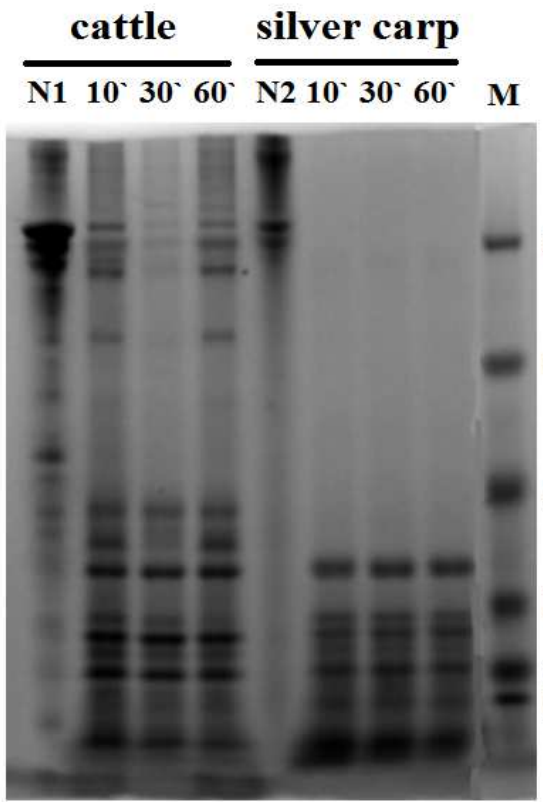

(A)
Trypsin

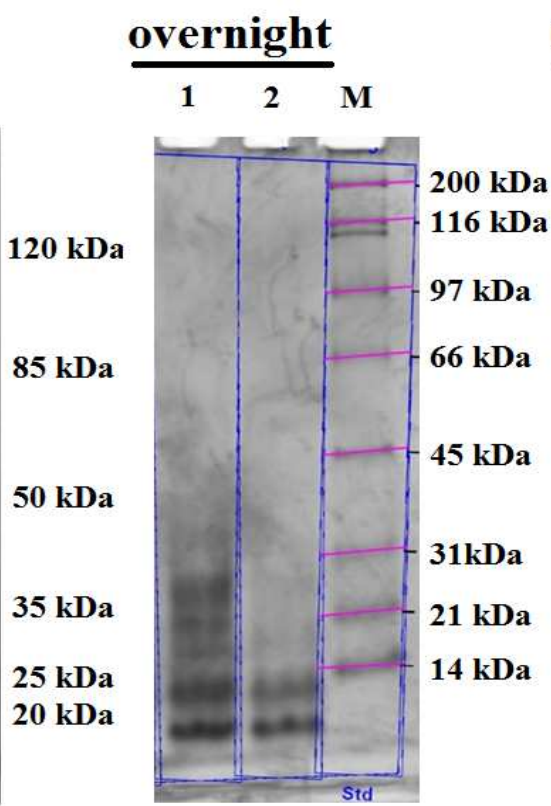

(B)
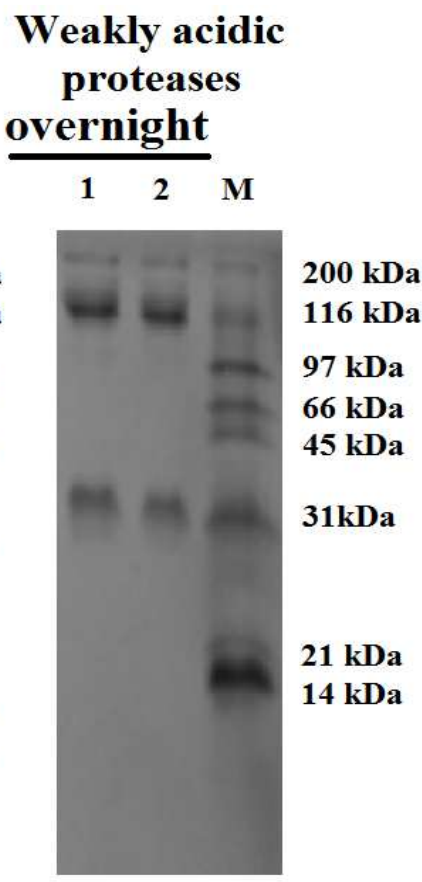

(C)

Figure 1 SDS-PAGE analysis of collagens from different organisms before and after proteolytic digestion. A) Products of digestion with a collagenase during 10, 30 and $60 \mathrm{~min}$.; B) Products of trypsinolysis: 1. Collagen from silver carp, 2. Collagen from cattle; C) Products of digestion with a mixture of weakly acidic proteases: 1 . silver carp collagen, 2 . cattle collagen

MALDI-TOF MS-spectroscopic analysis of proteolytic digestion products. We evaluated the molecular profiles of peptides after proteolytic digestion of collagens from silver carp and cattle by three proteases by MALDI-TOF massspectroscopy. As we can observe, Collagen from a silver carp (Figure 2 A-C) produces more complex profiles of peaks corresponding to proteolytic peptides appearing upon digestion by any of the three proteases than does Collagen from a cattle (compare Figure 2 D-F). Treatment of both collagen samples with a mixture of weakly acidic 
proteases gives a similar profile of peptides with molecular weights of 481, 576, 916, 1493, 2231, 2554, 3153, and $3808 \mathrm{Da}$. However, two peptides are missing in the case of cattle collagen digestion with a mixture of weakly acidic proteases. The cattle collagen digested with a mixture of weakly acidic proteases gives 481, 916, 1493, 2231, 2554, and 3907 Da peptides. Treatment of silver carp collagen solution with collagenase gives peptides with molecular weights of 481, 916, 1493, 2231, 2554 and $3808 \mathrm{Da}$. The same treatment of cattle collagen solution gives a pattern of peaks corresponding to $567,916,2666,4148$ and 5119
Da peptides. Mass-spectroscopic analysis of tryptic digestion products of two collagens demonstrates differences in sensitivity of two collagens to trypsin. There were 481, 1654, 2231, 2554, and 3808 Da peptides detected in the fish collagen solution, and 567, 1042, 2599, and 5422 Da peptides in the mammalian collagen solution. There were 481, 2231, 2554 and $3808 \mathrm{Da}$ peptides detected in the fish collagen solution after treatment with all three types of proteases which we used as a representative. No similar pattern was detected for the cattle collagen solution.

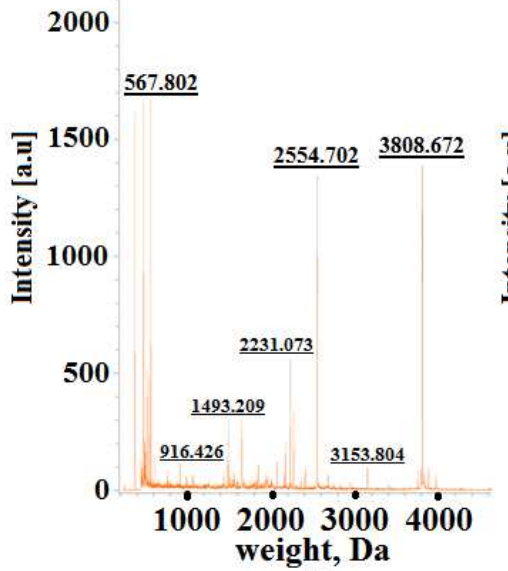

(A)

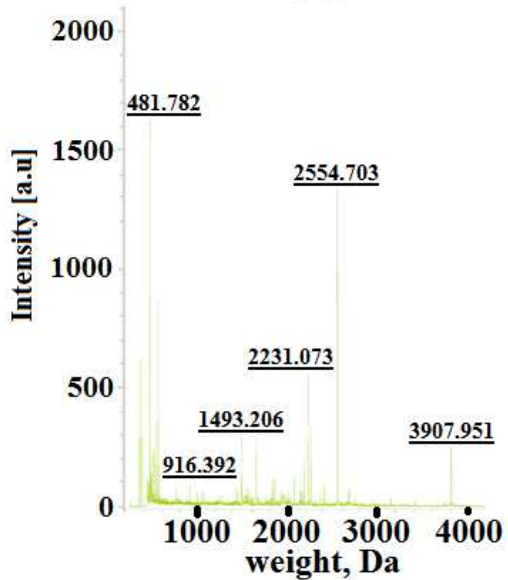

(D)

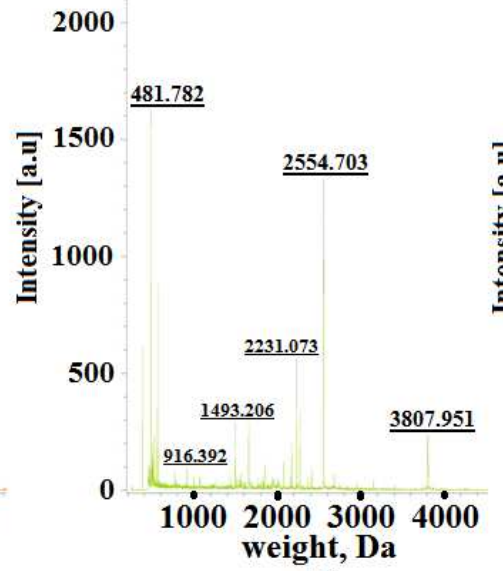

(B)

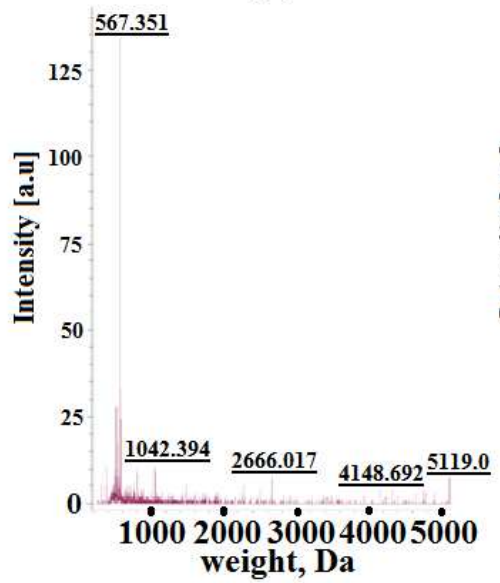

(E)

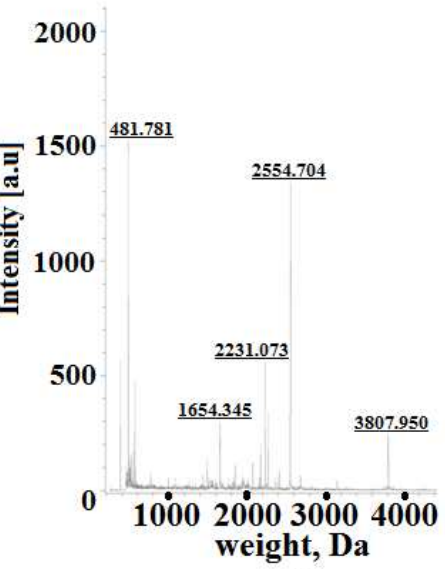

(C)

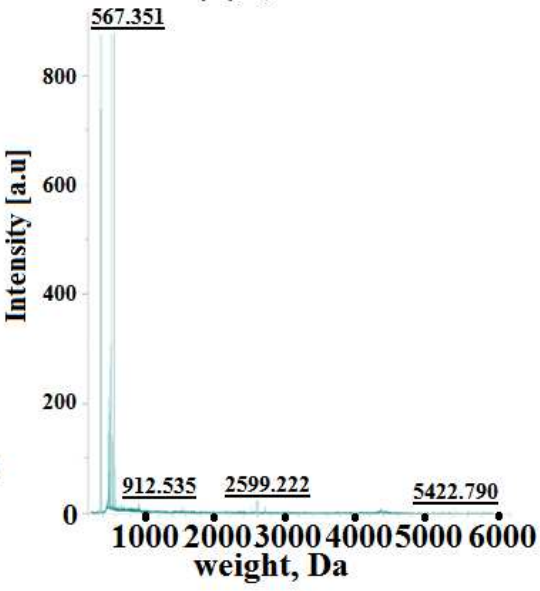

(F)

Figure 2 MALDI-TOF mass-spectrogram of silver carp collagen (A, B, C) and cattle collagen (D, E, F) proteolytic digestion products by A, D - a mixture of weakly acidic proteases; $\mathrm{B}, \mathrm{E}$ - collagenase; $\mathrm{C}, \mathrm{F}-$ trypsin

\section{CONCLUSION}

We have compared products of proteolytic digestion of collagens from a mammal organism (Bos taurus taurus) and a hydration organism (Hypophthalmichthys molitrix) upon digestion with different proteases (a mixture of weakly acidic proteases, trypsin and collagenase) by two different assays (SDS-PAGE and MALDI TOF spectrometry). Employment of these two assays allowed comparing peptides with high (in $\mathrm{kDa}$ range) and low (in Da range) molecular masses. We summarized the observed profiles of peptides in-Table 1. The hydration Collagen is susceptible to hydrolysis by any of the three proteolytic enzymes in varying degrees. This fact may reflect a higher content of arginine and lysine residues in the hydration collagen sequence, as previously suggested [16]. The rate of hydrolysis by collagenase and trypsin in the case of collagens from fish is higher compared to mammalian collagens. This fact may suggest a lower degree of packing of the macromolecular chains of hydration Collagen, 
which would make them more accessible to proteases. The obtained results imply a unique structure and novel physicochemical properties (surface charge and dipole

Table 1 Approximate molecular weight of collagen peptides during proteolytic digestion as estimated by SDS- moment) [17] of Collagen from hydrations and require further investigation.

PAGE and MALDI-TOF assays

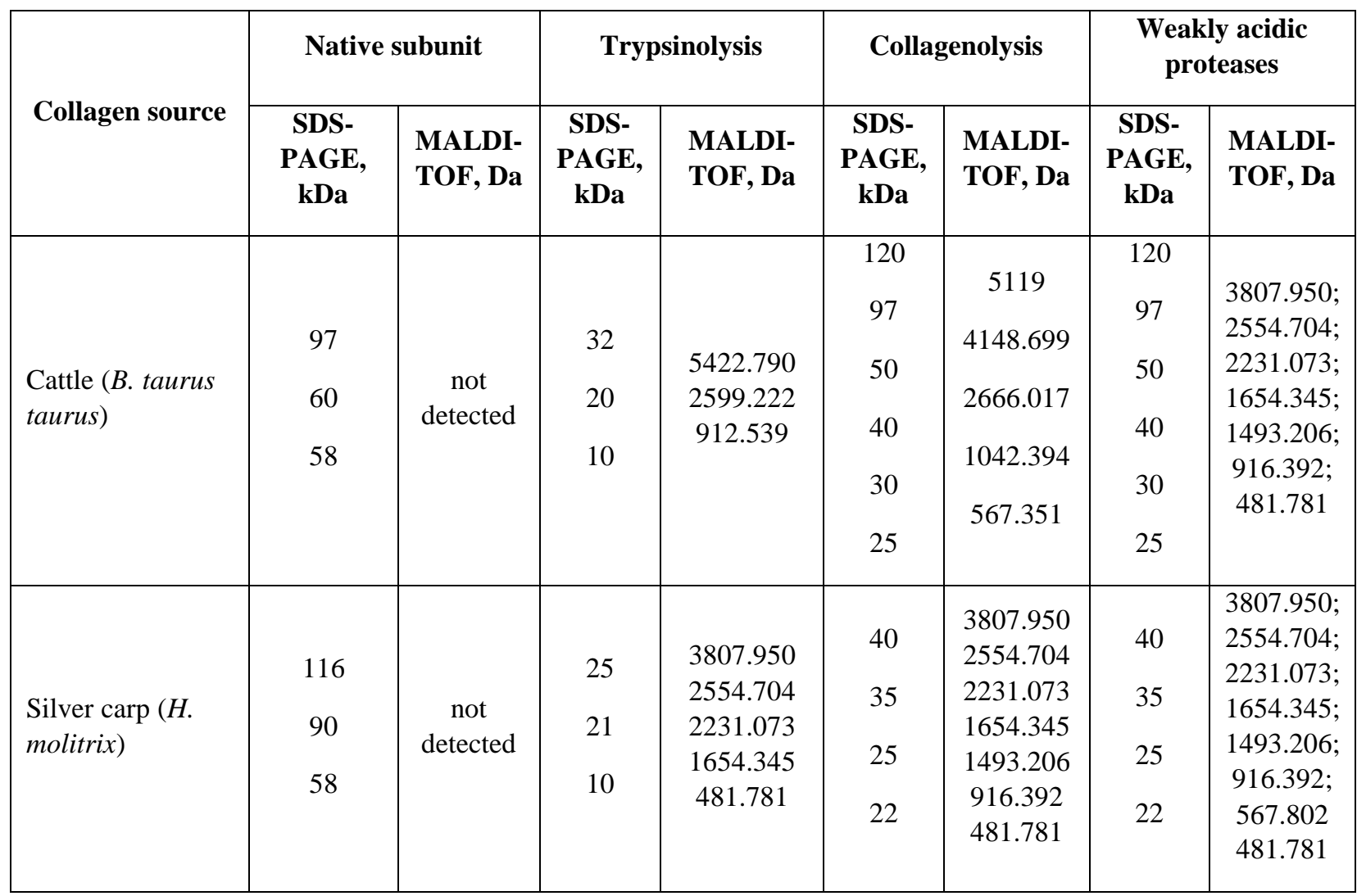

Interestingly, the products of collagen hydrolysis are different when treated with trypsin and collagenase, while a mixture of weakly acidic proteases gives a similar peptide composition for both collagens. We can speculate that in the case of the silver carp collagen, the amino acid sequence is as follows Gly-Pro-Gly-Hyp-Gly-Pro-HypGly-Gly-Lys (938.99 Da), which is formed by two fragments with a molecular weight $\sim 481 \mathrm{Da}$. The masses of the peptides are determined by the frequency of lysine or arginine residues occurrence [18]. The more lysine or arginine residues in the collagen molecule, the more shortchain peptides is observed in the hydrolysis products. While collagens hydrolysis with a mixture of weakly acidic proteases, allow suggesting that the amino acids of cattle and silver carp collagens, which are sensitive to these enzymes, are similar and organized similarly. Based on the analysis of the amino acid composition of collagen Type 1, we suggest that the amino acids sensitive to acidic proteases aspartic and glutamic acids. Thus, silver carp collagen may be cleaved by the proteases due to the presence of the following peptide sequence:

(-Ala-Gly-Pro-Gly-Hyp-Gly-Pro-Hyp-Gly-Gly-Lys-Asp-)

While cattle collagen is cleaved by the proteases due to the following peptide sequence:
(-Ala-Gly-Pro-Gly-Hyp-Gly-Pro-Hyp-Gly-Gly-Asp-) $)_{\mathrm{n} 1}-$ Lys-) ${ }_{\mathrm{n} 2}$ The difference in the molecular weight profiles of cattle and fish collagen peptides are significant while undigested collagens have almost the same molecular weight. It is known that collagens isolated from the skin of various organisms have different sensitivity to trypsin. This can be explained by the frequency of occurrence of lysine and arginine residues in collagens sequences. For example, Collagen from squid has many lysine and arginine residues, so it is cleaved very often by trypsin to produce small $(10 \mathrm{kDa})$ molecules suitable for secretion [19]. For fish and mammalian collagens, it is known that they are differentially enriched in proline, valine, methionine and tyrosine amino acids. These differences in the amino acid content may affect protease resistance of these collagens. We can conclude that it is possible to get Collagen from the fish skin according to our protocol, as the purified substance is specifically cleaved by collagenase. We compared the sensitivity of fish and cattle collagens to trypsin, collagenase and a mixture of weakly acidic proteases. We compared the molecular profiles of the collagen peptides upon proteolytic cleavage with different proteases. In conclusion, the obtained structural and biochemical data is a good starting point for further 
fundamental and applied research in the field of physiology, biotechnology, and biochemistry of Collagen [20].

\section{ACKNOWLEDGMENTS}

This research was supported by the Russian Academic Excellence Project at the Immanuel Kant Baltic Federal University.

\section{REFERENCES}

[1] L.V. Antipova, S.A. Storublevtsev, S.S. Antipov, Collagen: sources, properties, application, GIORD Publ., 2019.

[2] M.E. Uspenskaya, L.V. Antipova, M.I. Chubirko et al., Gigiena i sanit. 94(9) (2015) 47-50.

[3] S. Ricard-Blum, The Collagen Family, Cold Spring Harbor Perspect. in Biol. 3(1) (2011) a004978.

[4] S.P. Gabuda, A.A. Gaǐdash, E.A. Viazovaia, Biofizika 50(2) (2005) 231-235.

[5] A.A. Jalan, D. Sammon, J.D. Hartgerink et al., Nature Chem. Biol. (2020). Retrieved from: https://doi.org/10.1038/s41589-019-0435-y

[6] Y. Zhao, T.C. Marcink, R.R. Sanganna et al., Structure 23(2) (2015) 257-269.

[7] G.Y. Li, J. of Amer. Leather Chem. Associat. 98 (2003) 224-229.
[8] A.V. Finkelstein, Physics of Protein molecules, Inst. for Computer Res. Publ., 2014.

[9] L.V. Antipova, S.A. Storublevtsev, A.A. Getmanova, Proc. of the Voronezh State Univer. of Engineer. Technol. 80(3) (2018) 97-103.

[10] V.G. Lobanov, G.I. Kasyanov, E.A. Mazurenko, Proc. of the Voronezh State Univer. of Engineer. Technol. 81(1) 2019 160-167.

[11] L.J. Gould, Advan. in Wound Care 5(1) (2016) 19-31.

[12] I.A. Glotova, V. Boltachev, Advan. in Modern Natural Sci. 2 (2008) 43-44.

[13] P. Szpak, J. of Archaeolog. Sci. 38(12) (2011) 33583372.

[14] R.L. Gundry, M.Y. White, C.I. Murray et al., Current Protocols in Molec. Biol. 90(1) (2010) 10.25.1-10.25.23.

[15] U.K. Laemmli, Nature 227(5259) (1970) 680-685.

[16] B. Lin, F. Zhang, Y. Yu et al., The British J. of Nutrit. 107 (2011) 1160-1166.

[17] F. Ascencio, G. Johansson, T. Wadström, Archives of Microbiol. 164(3) (1995) 223-230.

[18] F. Ortolani, M. Giordano, M. Marchini, Biopolymers 54(6) (2000) 448-463.

[19] A. Veeruraj, M. Arumugam, T. Ajithkumar, T.Balasubramanian, Food Hydrocolloids 43 (2015) 708e716-716.

[20] S. Storublevtsev, L. Antipova, L. Matskova, FEBS Open Bio 9(Suppl. 1) (2019) 65-431. 